This item was submitted to Loughborough's Research Repository by the author.

Items in Figshare are protected by copyright, with all rights reserved, unless otherwise indicated.

\title{
Shear-free relativistic fluids and the absence of movable branch points
}

PLEASE CITE THE PUBLISHED VERSION

LICENCE

CC BY-NC-ND 4.0

\section{REPOSITORY RECORD}

Halburd, R.G.. 2019. "Shear-free Relativistic Fluids and the Absence of Movable Branch Points". figshare. https://hdl.handle.net/2134/468. 


\title{
Shear-Free Relativistic Fluids and the Absence of Movable Branch Points
}

\author{
R.G. Halburd \\ Department of Mathematical Sciences \\ Loughborough University \\ Loughborough, Leics, LE11 3TU, UK \\ R.G.Halburd@lboro.ac.uk
}

Short title: Shear-free relativistic fluids.

19 November 2001

(To appear in the Journal of Mathematical Physics)

\begin{abstract}
The problem of determining the metric for a non-static shear-free spherically symmetric fluid (either charged or neutral) reduces to the problem of determining a one parameter family of solutions to a second-order ODE containing two arbitrary functions $f$ and $g$. Choices for $f$ and $g$ are determined such that this ODE admits a one-parameter family of solutions that have poles as their only movable singularities. This property is strictly weaker than the Painlevé property and it is used to identify classes of solvable models. It is shown that this procedure systematically generates many exact solutions including the Vaidya metric, which does not arise from the standard Painlevé analysis of the second-order ODE. Interior solutions are matched to exterior Reissner-Nordstrøm metrics. Some solutions given in terms of second Painlevé transcendents are described.
\end{abstract}




\section{Introduction}

Several authors have shown that the problem of finding a non-static solution of the Einstein-Maxwell equations for a shear-free spherically symmetric charged fluid is equivalent to the problem of finding a $t$-dependent solution to the equation

$$
\frac{\partial^{2} y(x, t)}{\partial x^{2}}=f(x) y^{2}(x, t)+g(x) y^{3}(x, t)
$$

where $f$ and $g$ are arbitrary functions of $x$ only [1, 2] (see also [3] and the references therein). Given a solution $y$ of equation (1), define $r=\sqrt{x}$, $Y(r, t)=1 / y(x, t)$, and

$$
T(r, t)=h(t) \frac{\partial}{\partial t} \ln y\left(r^{2}, t\right)
$$

where $h$ is an arbitrary non-vanishing function of $t$. In terms of these variables, the metric for the fluid is given by

$$
d s^{2}=T^{2}(r, t) d t^{2}-Y^{2}(r, t)\left\{d r^{2}+r^{2} d \Omega^{2}\right\},
$$

where $d \Omega^{2}=d \theta^{2}+\sin ^{2} \theta d \phi^{2}$ is the standard metric on the 2-sphere. The density $\rho$ and pressure $p$ are given by

$$
\begin{aligned}
& 8 \pi \rho=3 h^{-2}-12 x y_{x}^{2}+12 y y_{x}+8 x f y^{3}+6 x g y^{4} \\
& 8 \pi p=4 y\left(y-2 x y_{x}\right) \frac{y_{x t}}{y_{t}}+12 x y_{x}^{2}-8 y y_{x}+2 x g y^{4}-2 h^{-3} h_{t} \frac{y}{y_{t}}-3 h^{-2} .(5)
\end{aligned}
$$

The only non-vanishing components of the electromagnetic field are

$$
F_{01}=-F_{10}=-h(t) E(r) \frac{\partial y}{\partial t}
$$

where $E^{2}(r)=2 x g(x)$.

Although $y$ is a function of two variables $x$ and $t$, equation (1) is essentially an ODE for $y$ as a function of $x$. As an ODE, the general solution of equation (1) contains two arbitrary constants. The general solution of equation (1) viewed as a PDE is obtained by replacing these arbitrary constants with arbitrary functions of $t$. Since $T$ is a metric coefficient, it cannot be identically zero, so from equation (2) we see that $y$ must have a non-constant 
$t$-dependence. This leads us to the problem of finding families of solutions to equation (1) viewed as an ODE which depend on (at least) one parameter.

The connection between integrable systems (equations that are solvable, either explicitly or via a related linear problem) was first used by Kowlevskaya in her work on spinning tops $[4,5]$. She considered the equations of motion for a spinning top which depend on six parameters (the center of mass and the moments of inertia). Kowalevskaya noticed that in the known cases for which the equations could be integrated, the general solution was a meromorphic function of time when extended to the complex plane. She used local series analysis to determine all choices of the parameters for which the general solution was a meromorphic function of time and found a new set of values for the parameters for which she was then able to solve the equations in terms of ratios of hyper-elliptic functions.

The requirement that all solutions are meromorphic throughout the complex plane may be replaced with the requirement that all solutions be meromorphic on the covering space of $\mathbf{C}$ with a discrete set of points removed. In this way, branching of solutions is allowed at fixed singularities (singularities of the solutions that cannot occur at arbitrary locations in the complex plane but only at locations at which the equation itself is in some sense singular). An ODE is said to possess the Painlevé property if all movable singularities of all solutions are poles. This property is closely connected with the integrability (solvability) of the ODE. All ODEs that are known to possess the Painlevé property are integrable, either explicitly in terms of classically known functions, or via an associated linear problem. In particular, Painlevé, Gambier, and Fuchs classified all equations of the form

$$
\frac{d^{2} y}{d x^{2}}=F\left(x ; y, \frac{d y}{d x}\right),
$$

where $F$ is rational in $y$ and $d y / d x$ and analytic in $x$, that have the Painlevé property. They showed that each such equation could be transformed via a change of independent variable and an $x$-dependent Möbius transformation of $y$ to one of fifty canonical equations. With the exception of six equations (the Painlevé equations $P_{I}-P_{V I}$ ) each of these canonical equations were solved in terms of classically known functions (see, e.g., $[6,7]$ ). The first two Painlevé equations are

$$
\frac{d^{2} \eta}{d \zeta^{2}}=6 \eta^{2}+\zeta
$$




$$
\frac{d^{2} \eta}{d \zeta^{2}}=2 \eta^{3}+\zeta \eta+\alpha
$$

where $\alpha$ is an arbitrary complex constant. It was later shown that each Painlevé equation is the compatibility condition for a (linear) spectral problem. The Painlevé equations are considered to be integrable because of the underlying structure that emerges from these isomonodromy problems $[8,7]$.

It is important to note that the transformation of one the equations of the form (6) that possesses the Painlevé property to one of the canonical forms is itself determined by the solutions of a system of differential equations. A weaker definition of the Painlevé property is that all solutions are singlevalued about all movable singularities, however, for equations of the form (6) this definition yields the same class of equations.

Shah and Vaidya [1], Wyman [9, 10], Chatterjee [11], Maharaj, Leach, and Maartens [12] and Srivastava [13] have studied equation (1) to determine choices of $f$ and $g$ for which the general solution has no movable critical points. In particular, Wyman [9] determined all choices of $f$ in the uncharged $(g=0)$ case. In [14] the author found all choices of $f$ and $g$ such that equation (1) possesses the Painlevé property. In particular, we have the following.

\section{Proposition 1.1}

Equation (1) possesses the Painlevé property (as an ODE in x) if and only if either

1.

$$
f(x)=6 w^{5}(z), \quad g(x)=0,
$$

where $w \neq 0$ and $v$ are any solutions of

$$
\begin{aligned}
\frac{d^{2} v}{d z^{2}} & =6 v^{2}+a z+b / 2 \\
\frac{d^{2} w}{d z^{2}} & =12 v w
\end{aligned}
$$

where $a, b$ are constants and $z$ is given by

$$
x=\int w^{-2}(z) d z
$$

or 
2.

$$
f(x)=6 v(z) w^{5}(z), \quad g(x)=2 w^{6}(z),
$$

where $w \neq 0$ and $v$ are any solutions of

$$
\begin{aligned}
\frac{d^{2} v}{d z^{2}} & =2 v^{3}+(a z+b) v+c / 2, \\
\frac{d^{2} w}{d z^{2}} & =\left(6 v^{2}+a z+b\right) w,
\end{aligned}
$$

where $a, b, c$ are constants and $z$ is given by equation (12).

Furthermore, in both the above cases, the general solution of equation (1) is given by

$$
y(x, t)=\frac{u(z, t)-v(z)}{w(z)},
$$

where $u$ (in which $t$ is treated as a parameter) is the general solution of the same second-order equation as $v$ (i.e. in case $1, u\left(z, t_{0}\right)$, where $t_{0}$ is a constant, solves equation 10 and in case 2 it solves equation 14).

Note that equation (11) (resp. 15) is the linearization of equation (10) (resp. 14). So if $v(z)=V(z ; \epsilon)$ is a one-parameter family of solutions to equation (10) (resp. 14) then $w(z):=V_{\epsilon}(z ; \epsilon)$ is a solution to equation (11) (resp. 15). A second independent solution to equation (11) (resp. 15) then follows by reduction of order. Equation (12) shows that $x=\hat{w}(z) / w(z)$, where $\hat{w}$ is a second solution of equation (11) (resp. 15) satisfying the Wronskian condition $W(w, \hat{w})=w \hat{w}_{z}-\hat{w} w_{z}=1$.

If $a=0$ then the general solution to equation (10) (resp. 14) can be given explicitly in terms of elliptic functions. In particular, the case in which $a=0$ and the fixed solution $v$ of equation (14) is a constant corresponds to the large class of solutions found by Sussman [15]. In fact, most of the solutions that have appeared in the literature to date are special cases of Sussman's solutions. The case in which $v$ is not constant is solved explicitly in [14]. If $a \neq 0$ then equation (10) (resp. 14) can be mapped to equation (7) (resp. 8). A class of solutions to equation (1) corresponding to the Airy function solutions to equation (8) are also described in [14].

Recall that we wish to find one-parameter families of solutions to equation (1). When $f$ and $g$ are chosen so that equation (1) possesses the Painlevé 
property then the equation is integrable and we can find a two-parameter family of solutions. In the present paper a property, weaker than the Painlevé property but still complex-analytic in nature, is considered. Namely, we wish to find all one-parameter families of solutions $\mathcal{F}$ to equation (1) such that all movable singularities of all solutions in $\mathcal{F}$ are poles. In section 2 we will find all solutions to equation (1) that are simultaneously solutions of a Riccati equation. This class of solutions contains the well-known solutions due to Shah and Vaidya [16], which does not arise in a regular Painlevé analysis of equation (1). A class of solutions that generalizes that due to Shah and Vaidya which is given in terms of solutions to linear equations is also derived.

Sections 3 and 4 address the question of whether the solutions found in section 2 exhaust the set of all one-parameter families of solutions $\mathcal{F}$ described above. In section 5 , boundary conditions are determined such that the Riccati solutions can be matched to the Reissner-Nordstrøm external solution. In section 6 we find solutions to equation (1) corresponding to $a \neq 0$ but $v \equiv 0$ in equation (14). In this case the general solution to equation (15) is given in terms of Airy functions. From this solution, families of solutions are obtained using the Bäcklund transformation of the second Painlevé equation.

\section{Riccati solutions}

One way of finding a one-parameter family of solutions to equation (1) such that the only movable singularities are poles is to find a family of solutions that are also solutions of a first-order equation of Painlevé type. In this section, solutions to equation (1) are found that are also solutions to a firstorder differential equation of the form

$$
\frac{d y}{d x}=R(x, y)
$$

where $\mathrm{R}$ is rational in $y$ and locally analytic in $x$. Fuchs [17] showed that the only equations of the form (17) with the Painlevé property is the Riccati equation,

$$
\frac{d y}{d x}=\alpha(x) y^{2}+\beta(x) y+\gamma(x)
$$

where $\alpha, \beta$, and $\gamma$ are (locally) analytic functions of $x$. The general solution of equation (18) is given by

$$
y(x)=-\frac{1}{\alpha(x)} \frac{d}{d x} \ln \Phi(x),
$$


where $\Phi$ is the general solution of the linear equation

$$
\frac{d^{2} \Phi}{d x^{2}}-\left(\beta+\frac{\alpha_{x}}{\alpha}\right) \frac{d \Phi}{d x}+\alpha \gamma \Phi=0 .
$$

Differentiating equation (18) with respect to $x$ and again using equation (18) to eliminate $d y / d x$ in the resulting expression gives

$$
\frac{d^{2} y}{d x^{2}}=2 \alpha^{2} y^{3}+\left(\alpha_{x}+3 \alpha \beta\right) y^{2}+\left(\beta_{x}+\beta^{2}+2 \alpha \gamma\right) y+\left(\gamma_{x}+\beta \gamma\right) .
$$

It follows that every solution of equation (18) is a solution of equation (1) if and only if the equations

$$
\begin{aligned}
\gamma_{x}+\beta \gamma & =0 \\
\beta_{x}+\beta^{2}+2 \alpha \gamma & =0 \\
\alpha_{x}+3 \alpha \beta & =f \\
2 \alpha^{2} & =g
\end{aligned}
$$

are satisfied.

Solving equations (21-24) gives three classes of Riccati equations.

Case 1: $\beta \equiv 0, \gamma \equiv 0$. The Riccati equation (18) becomes

$$
\frac{d y}{d x}=\alpha y^{2},
$$

which has the general solution

$$
y(x, t)=\frac{1}{H(x)+G(t)},
$$

where $H^{\prime}(x)=-\alpha(x)$ and $G$ is an arbitrary function of $t$.

Case 2: $\beta \not \equiv 0, \gamma \equiv 0$. The Riccati equation (18) becomes

$$
\frac{d y}{d x}=\alpha y^{2}+\frac{1}{x+C} y
$$

where $C$ is an arbitrary constant, which has the general solution

$$
y(x, t)= \begin{cases}\frac{x}{H(x)+G(t)}, & H^{\prime}(x)=-x \alpha(x), \text { if } C=0, \\ \frac{1+k x / 4}{H(x)+G(t)}, & H^{\prime}(x)=-(1+k x / 4) \alpha(x), \text { if } C=4 / k \neq 0\end{cases}
$$


where $G$ is an arbitrary function of $t$.

Case 3: $\gamma \not \equiv 0$. The Riccati equation (18) becomes

$$
\frac{d y}{d x}+\frac{1}{2}\left(\gamma^{-1}\right)_{x x} y^{2}+\frac{\gamma^{\prime}}{\gamma} y-\gamma=0
$$

In section 5, these Riccati solutions will be matched to an external ReissnerNordstrøm metric.

Note that equation (25) corresponds to setting $k=0$ in equation (26b). Under the transformation

$$
\tilde{r}=\frac{r}{1+k r^{2} / 4}
$$

(recall $x=r^{2}$ ) the solutions corresponding to equation (26b) give rise to the metric

$$
d s^{2}=[F(\tilde{r})+G(t)]^{-2} d t^{2}-[F(\tilde{r})+G(t)]^{2}\left[\frac{d \tilde{r}^{2}}{1-k \tilde{r}^{2}}+\tilde{r}^{2} d \Omega^{2}\right],
$$

where $F(\tilde{r})=H\left(r^{2}\right)$ and we have set $h(t)=1 / \dot{G}(t)$. The metric (28) was obtained by Shah and Vaidya [16]. This metric does not arise from the standard Painlevé analysis of equation (1).

Solutions found in this section will be referred to as Riccati solutions.

\section{Local series analysis}

In this section we will analyze equation (1) as an ODE in the complex domain. In particular, we will determine necessary conditions that equation (1) possesses a one-parameter family of Laurent series solutions. We begin by considering the case in which $g=0$. Under the transformation (16) in which $w$ is given by equation (9), $v$ is given by equation (11), and $z$ is given implicitly by equation (12), equation (1) becomes

$$
\frac{d^{2} u}{d z^{2}}=6 u^{2}+A(z)
$$

where

$$
A(z)=\frac{d^{2} v}{d z^{2}}-6 v^{2}
$$


We will only consider a one-parameter family of solutions $\mathcal{G}$ such that there exists an open connected set $\Omega \in \mathbf{C}$ such that at each point $z_{0} \in \Omega$ there is a function $u \in \mathcal{G}$ with a pole at $z=z_{0}$. We will now find a necessary condition on the function $A$ such that equation (29) admits a formal Laurent series solution with a pole at a point $z_{0} \in \Omega$, where $A$ is analytic. Substituting the Laurent series

$$
u(z)=\sum_{n=0}^{\infty} a_{n}\left(z-z_{0}\right)^{n-p},
$$

where $p$ is a positive integer and $a_{0} \neq 0$, into equation (29) gives, to leading order,

$$
p(p+1) a_{0}\left(z-z_{0}\right)^{-(p+2)}+\cdots=6 a_{0}^{2}\left(z-z_{0}\right)^{-2 p}+\cdots .
$$

Equating the powers and coefficients of these leading-order terms gives

$$
p=2, \quad a_{0}=1 .
$$

Using equations (30) and (31) in equation (29) and equating coefficients of like powers of $z-z_{0}$ gives

$$
(n+1)(n-6) a_{n}=P_{n}\left(a_{0}, a_{1}, \ldots, a_{n-1}\right),
$$

where

$$
P_{n}\left(a_{0}, a_{1}, \ldots, a_{n-1}\right)=6 \sum_{m=1}^{n-1} a_{m} a_{n-m}+\alpha_{n}\left(z_{0}\right),
$$

and

$$
\alpha_{n}\left(z_{0}\right)= \begin{cases}0, & n<4, \\ \frac{A^{(n-4)}\left(z_{0}\right)}{(n-4) !}, & n \geq 4,\end{cases}
$$

is polynomial in its arguments and we have expanded $A$ as a power series about $z=z_{0}$. The recurrence relation (32) shows that $a_{n}$ is uniquely determined in terms of $\left\{a_{0}, \ldots, a_{n-1}\right\}$, except in the case when $n=6$. In this case, the left side of equation (32) vanishes while the right side is a known function of $\left(a_{0}, \ldots, a_{5}\right)$. If the right side does not vanish, then there is no solution to equation (29) with a pole of any order at $z=z_{0}$. If the right side of equation (32) does vanish then a formal Laurent series solution exists in which $z_{0}$ and $a_{6}$ are arbitrary constants. A direct calculation shows that the right side of equation (32) vanishes if and only if

$$
A^{\prime \prime}\left(z_{0}\right)=0 .
$$


Now since equation (33) must be satisfied for all $z_{0}$ in the open set $\Omega$, this implies that $A(z)=a z+b / 2$, for some constants $a$ and $b$. So we reproduce precisely those solutions given in case 1 of Proposition 1.1.

Now we consider the local series analysis of equation (1) when $g$ is not identically zero. In particular, $g$ does not vanish identically on $\Omega$. From section 2 we see that the requirement that there is a one-parameter family of solutions such that all movable singularities are poles yields more solutions than requiring that equation (1) possesses the Painlevé property.

Let $v$ and $w$ be defined by equation (13) where $z$ is given by equation (12). (Note that we are not assuming that equations (14-15) hold.) The transformation (16) gives

$$
\frac{d^{2} u}{d z^{2}}=2 u^{3}+B(z) u+C(z)
$$

where

$$
B(z)=\frac{w_{z z}}{w}-6 v^{2}, \quad C(z)=v_{z z}-v B(z)-2 v^{3} .
$$

We now look for a local Laurent series solution to equation (34) with a pole at $z=z_{0} \in \Omega$. Leading order analysis shows that any such solution $u$ must have a simple pole at $z=z_{0}$ with residue \pm 1 . Hence we substitute the series

$$
u(z)=\sum_{n=0}^{\infty} a_{n}\left(z-z_{0}\right)^{n-1}, \quad a_{0}=\varepsilon= \pm 1
$$

into equation (34) and equate coefficients of like powers of $z-z_{0}$ to obtain the recurrence relation

$$
(n+1)(n-4) a_{n}=Q_{n}\left(a_{0}, \ldots, a_{n-1}\right),
$$

where

$$
Q_{n}\left(a_{0}, \ldots, a_{n-1}\right)=2\left[\sum_{m=0}^{n} \sum_{m^{\prime}=0}^{n-m} a_{m} a_{m^{\prime}} a_{n-m-m^{\prime}}-3 a_{n}\right]+\beta_{n}\left(z_{0}\right)+\gamma_{n}\left(z_{0}\right),
$$

and

$$
\beta_{n}\left(z_{0}\right)=\left\{\begin{array}{ll}
0, & n<2, \\
\sum_{m=0}^{n-2} \frac{B^{(n-m-2)}\left(z_{0}\right)}{(n-m-2) !} a_{m}, & n \geq 2,
\end{array} \quad \gamma\left(z_{0}\right)= \begin{cases}0, & n<3, \\
\frac{C^{(n-3)}\left(z_{0}\right)}{(n-3) !}, & n \geq 3 .\end{cases}\right.
$$


Note that Q is polynomial in its arguments. The left side of equation (35) shows that a necessary and sufficient condition for the existence of a formal Laurent series solution with a pole at $z=z_{0}$ is $Q_{4}\left(a_{0}, a_{1}, a_{2}, a_{3}\right)=0$, which is equivalent to $B^{\prime \prime}\left(z_{0}\right)=-2 \varepsilon C^{\prime}\left(z_{0}\right)$, where $\varepsilon= \pm 1=a_{0}$.

Now the general solution of equation (34) will have movable singularities with leading order behaviors that include both +1 and -1 residue poles (although, in general, these solutions will not be meromorphic and the Laurent series will have to be augmented by logarithm terms). So if we demand that all movable singularities of all solutions are poles (i.e., if we demand that equation (34) possess the Painlevé property) then $B^{\prime \prime}\left(z_{0}\right)=2^{\prime} C\left(z_{0}\right)$ and $B^{\prime \prime}\left(z_{0}\right)=-2 C^{\prime}\left(z_{0}\right)$, for all $z_{0} \in \Omega$, leading to $B(z)=a z+b$, and $C(z)=c / 2$, where $a, b$, and $c$ are arbitrary constants. So $u$ satisfies equation (14).

Rather than demand that all movable singularities of all solutions of equation (34) are poles, we restrict our consideration to a subset of solutions $\mathcal{G}$ such that given any $z_{0} \in \Omega$, there is a solution in $\mathcal{G}$ with a pole at $z=z_{0}$. The above analysis shows that either we are left with equation (14) or we must consider the class of solutions where all movable singularities are poles with the same residue $\varepsilon= \pm 1$. A necessary condition in this case is the differential equation $B^{\prime \prime}(z)=-2 \varepsilon C^{\prime}(z)$. In terms of $q(z):=B(z) / 2$, we now restrict ourselves to the study of the subset of solutions to the equation

$$
\frac{d^{2} u}{d z^{2}}=2 u^{3}+2 q u+\left(\kappa-\varepsilon q_{z}\right), \quad \varepsilon= \pm 1,
$$

where $\kappa$ is an arbitrary constant, that admit only poles with residue $\varepsilon$ in $\Omega$.

\section{The uniqueness of the Riccati solutions}

The only Riccati equation for which all solutions $u$ are also solutions of equation (36) is

$$
\frac{d u}{d z}+\varepsilon\left(u^{2}+q\right)=0, \quad \kappa=0 .
$$

The general solution of equation (37) is given by

$$
u=\varepsilon \frac{d}{d z} \ln \Phi
$$

where $\Phi$ is the general solution of the linear equation

$$
\frac{d^{2} \Phi}{d z^{2}}+q \Phi=0
$$


We will show that these Riccati type solutions are identical to those found in section 2. All movable singularities of any solution to equation (37) are simple poles with residue $\varepsilon$. So the general solution to equation (37) is a one-parameter family of solutions to equation (36) of the kind considered at the end of the previous section. The perturbation argument described below suggests that this is the only such one-parameter family. We will then provide a proof based on Wiman-Valiron theory for the case in which $q$ is a polynomial. Wiman-Valiron theory is particularly useful for finding entire solutions of analytic differential equations [18].

We will now show how the Riccati equations derived in section 2 are related to the solutions of equation (36) described at the end of section 3 . It may be verified that the identity

$$
w\left(u_{z}+\varepsilon\left[u^{2}+q\right]\right)=y_{x}+\varepsilon w^{3} y^{2}+w\left(w_{z}+2 \varepsilon v w\right) y+w\left(v_{z}+\varepsilon\left[v^{2}+q\right]\right)
$$

follows from equations (12) and (16). Furthermore, given equations (12) and (13), where $v$ and $w$ satisfy

$$
\begin{aligned}
v_{z z} & =2 v^{3}+2 q v-\varepsilon q_{z}, \quad \text { and } \\
w_{z z} & =\left(6 v^{2}+2 q\right) w,
\end{aligned}
$$

respectively, it can be shown that equations (21-24) are equivalent to

$$
\begin{aligned}
\alpha & =-\varepsilon w^{3}, \\
\beta & =-w\left(w_{z}+2 \varepsilon v w\right), \\
\gamma & =-w\left(v_{z}+\varepsilon\left[v^{2}+q\right]\right) .
\end{aligned}
$$

Equations (40) and (43-45) show that the Riccati solutions found in section 2 are the same as those constructed using (16) where $u$ is the general solution of equation (37) and $v, w$ satisfy equations (41) and (42) respectively. It is interesting to note that the solution by Shah and Vaidya discussed in section 2 corresponds to the case in which $v$ also satisfies the Ricatti equation (37). The $\gamma \neq 0$ case (case 3 in section 2), corresponds to a non-Ricatti solution $v$ of equation (41) (although $u$ still satisfies a Riccati equation).

Next we address the question of whether the class $\mathcal{G}$ consists only of Riccati solutions. Consider equation (36) with $q(z)=q_{0}+h Q(z)$, where $q_{0}$ is a complex constant and $h$ is a small complex parameter. To leading order in $h$, equation (36) is

$$
\frac{d^{2} u}{d z^{2}}=2 u^{3}+2 q_{0} u+\kappa
$$


If $u$ is not constant then

$$
\left(\frac{d u}{d z}\right)^{2}=u^{4}+2 q_{0} u^{2}+2 \kappa u+C,
$$

where $C$ is an integration constant. The non-constant solutions of equation (46) are elliptic functions with simple poles of residue \pm 1 . The only solutions with poles of residue $\varepsilon= \pm 1$ but no poles of residue $-\varepsilon=\mp 1$ correspond to the case in which $\kappa=0$ and $C=q_{0}^{2}$ in which case equation (46) factors into two Riccati equations and $u$ satisfies

$$
\frac{d u}{d z}+\varepsilon\left(u^{2}+q_{0}\right)=0 .
$$

The arguments given above assume that the one-parameter family of solutions $\mathcal{G}$ have poles in an open set $\Omega$. In the following we show rigorously that we have found all one-parameter families of solutions that have only poles as their movable singularities under the assumption that $q$ is a polynomial.

Consider the system of first-order equations

$$
\begin{aligned}
& \frac{d u}{d z}=\tilde{u}-\varepsilon u^{2}-\varepsilon q, \\
& \frac{d \tilde{u}}{d z}=\kappa+2 \varepsilon u \tilde{u} .
\end{aligned}
$$

Differentiating equation (47) with respect to $z$ and using equation (48) to eliminate $d \tilde{u} / d z$ gives equation (36). We wish to show that if there is a one-parameter family of solutions $u$ having only movable poles with residue $\varepsilon$ then $\tilde{u}$ is identically zero. Note that if $\tilde{u}$ is identically zero then equation (47) becomes equation (37) and equation (48) implies that $\kappa=0$. If $\tilde{u}$ does not vanish identically then we can solve equation (48) for $u$ and substitute it into equation (47) to give

$$
2 \tilde{u} \frac{d^{2} \tilde{u}}{d z^{2}}=\left(\frac{d \tilde{u}}{d z}\right)^{2}+4 \tilde{u}^{2}(\varepsilon \tilde{u}-q)-\kappa^{2} .
$$

We will prove the following.

Proposition 4.1 If $q$ is a polynomial then either any entire solution of equation (49) is a constant or

$$
q=q_{0}+q_{1} z+q_{2} z^{2}
$$


where

$$
q_{1}^{2}-4 q_{0} q_{2}=\kappa^{2}
$$

and

$$
\tilde{u}=\varepsilon q
$$

Note that if $\tilde{u}$ is one of the solutions given in Proposition 4.1 but $\tilde{u} \not \equiv 0$, then since $\tilde{u}$ contains no free parameters (i.e. no parameters other than those in the equation itself) $u$ is given by equation (48) and so does not represent a one-parameter family of solutions to equation (36).

\section{Proof}

We will begin by showing that any polynomial solution of equation (49) is either a constant or the solution (51). We will then use the central index from Wiman-Valiron theory to show that there are no transcendental (i.e. non-polynomial) solutions.

Let $q$ and $\tilde{u}$ be polynomials of degree $M$ and $N$ respectively. Furthermore we assume that $\tilde{u}$ is not constant (i.e. $N \geq 1$ and $\tilde{a}_{N} \neq 0$ ). Then $q$ and $\tilde{u}$ have expansions of the form

$$
q(z)=\sum_{m=0}^{M} q_{m} z^{m}, \quad \tilde{u}(z)=\sum_{n=0}^{N} \tilde{a}_{n} z^{n} .
$$

Substituting the expansions (52) into equation (49) and balancing the dominant terms for large $z$ gives $M=N$. Equation (49) then becomes

$$
\sum_{i, j=0}^{N} i(2 i-j-2) \tilde{a}_{i} \tilde{a}_{j} z^{i+j-2}+\kappa^{2}=\sum_{i, j, k=0}^{N} 4 \tilde{a}_{i} \tilde{a}_{j}\left(\varepsilon \tilde{a}_{k}-q_{k}\right) z^{i+j+k} .
$$

Now the polynomial on the left side of equation (53) is of degree at most $2 N-2$ while the degree of the polynomial on the right side is of degree at most $3 N$. Since $\tilde{a}_{N} \neq 0$ then the coefficient of $z^{3 N}$ in equation (49) gives $\tilde{a}_{N}=\varepsilon q_{N}$. Arguing by induction, equating the coefficients of $z^{3 M-1}, z^{3 M-2}, \ldots, z^{2 N+1}$ to zero gives $\tilde{a}_{N-n}=\varepsilon q_{N-n}, n=1, \ldots, N$. Hence $\tilde{u}=\varepsilon q$ and the right side of equation (53) vanishes identically. On equating all coefficients of powers of $z$ to zero on the left side of equation (49) we find that $N=2$ and $q_{0}, q_{1}$, and $q_{2}$ satisfy equation (50). 
Now we will use Wiman-Valiron theory to show that all entire solutions to equation (49) are polynomials. Since $\tilde{u}$ is entire it has an expansion of the form

$$
\tilde{u}(z)=\sum_{n=0}^{\infty} \tilde{a}_{n} z^{n}
$$

The central index $\nu(r, \tilde{u})$ is the greatest non-negative integer $m$ such that

$$
\left|\tilde{a}_{m}\right| r^{m}=\max _{n \geq 0}\left|\tilde{a}_{n}\right| r^{n} \text {. }
$$

Since $\tilde{u}$ is non-polynomial then $\nu(r, \tilde{u})$ is increasing, piecewise constant, rightcontinuous, and tends to $+\infty$ as $r \rightarrow+\infty$.

In terms of the central index we have the following lemma (see, e.g., Jank and Volkmann [19]).

Lemma 4.2 Let $\tilde{u}$ be a non-polynomial entire function, and $\nu=\nu(r, \tilde{u})$ be its central index. Let $0<\delta<1 / 4$ and $z$ be such that $|z|=r$ and

$$
|\tilde{u}(z)|>\nu(r, \tilde{u})^{-\frac{1}{4}+\delta} \max _{|z|=r}|\tilde{u}(z)|
$$

holds. Then there exists a set $F \subset \mathbf{R}$ of finite logarithmic measure, i.e., $\int_{F} d t / t<+\infty$ such that

$$
\tilde{u}^{(m)}(z)=\left(\frac{\nu(r, \tilde{u})}{z}\right)^{m}(1+o(1)) \tilde{u}(z)
$$

holds for all $m \geq 0$ and $r \notin F$.

Lemma 4.2 says that for all positive $r$ outside of the set $\mathrm{F}$ (which has finite logarithmic measure) then the estimate (55) is holds near the maximum of $|\tilde{u}|$ on the circle $|z|=r$ (where "near the maximum" means the set of $z$ satisfying equation 54 ).

Assume that there is a non-polynomial solution $\tilde{u}$ of equation (49). Applying the estimate (55) to equation (49) gives

$$
\left(\frac{\nu(r, \tilde{u})}{z}\right)^{2} \tilde{u}^{2} \sim 4 \varepsilon \tilde{u}^{3}
$$

Since $\nu(r, \tilde{u})$ grows much slower than $\tilde{u}$ [20], it follows that equation (56) cannot be balanced. Thus the only entire solutions to equation (49) are polynomials. 


\section{Boundary conditions for the Riccati solu- tions}

In this section we will match the Riccati solutions introduced in section 2 to an external Reissner-Nordstrøm metric

$$
d s^{2}=\hat{\Gamma} d \hat{t}^{2}-\hat{\Gamma}^{-1} d \hat{r}^{2}-\hat{r}^{2} d \Omega^{2},
$$

where $d \Omega^{2}$ is the standard metric on the 2-sphere and

$$
\hat{\Gamma}=1-\frac{2 m}{\hat{r}}+\frac{4 \pi e^{2}}{\hat{r}^{2}},
$$

and $m$ and $e$ are constants. Let $\Sigma_{0}$ be the interface $r=r_{0}$ between the two solutions. The two metrics (3) and (57) can be matched across $\Sigma_{0}$ provided

$$
\begin{aligned}
p\left(r_{0}, t\right) & =0, \\
g\left(r_{0}^{2}\right) & =2 \pi\left(\frac{e}{r_{0}^{3}}\right)^{2}, \\
2 m & =\left[\frac{4 \pi e^{2}}{r} y+\frac{r^{3}}{h^{2} y^{3}}+2 \frac{r^{2}}{y^{2}} y_{r}-\frac{r^{3}}{y^{3}} y_{r}^{2}\right]_{r=r_{0}},
\end{aligned}
$$

for all $t[2]$.

Equation (59) is equivalent to

$$
\alpha^{2}\left(r_{0}^{2}\right)=\pi\left(\frac{e}{r_{0}^{3}}\right)^{2} .
$$

Using equation (18) to eliminate $y_{x}$ and $y_{x t}=(2 \alpha y+\beta) y_{t}$ from equation (5) gives

$$
\begin{aligned}
8 \pi p= & 4\left\{\left(\left[\beta^{2}+2 \alpha \gamma\right) x-\beta\right) y^{2}+2 \gamma(2 \beta x-1) y+3 x \gamma^{2}\right\} \\
& -3 h^{-2}-2 h^{-3} h_{t} \frac{y}{y_{t}}
\end{aligned}
$$

Using equation (18) to eliminate $y_{r}=2 r y_{x}$ from equation (60) and using equation (62) in equation (58), we see that equations (58) and (60) are equivalent to

$$
\begin{gathered}
h^{-2}(t)=4\left[r^{2} \gamma+\gamma\left(2 r^{2} \beta-1\right) y+\left\{\left(\beta^{2}+2 \alpha \gamma\right) r^{2}-\beta\right\} y^{2}\right. \\
\left.+\left(\frac{m}{2 r^{3}}+2 r^{2} \alpha \beta-\alpha\right) y^{3}\right]_{r=r_{0}} .
\end{gathered}
$$




\section{Dust solutions}

Setting $p$ identically zero in equation (62) and solving for $h^{-2}$ gives

$$
h^{-2}=4\left[x \gamma+\gamma(2 x \beta-1) y+\left\{\left(\beta^{2}+2 \alpha \gamma\right) x-\beta\right\} y^{2}+\delta y^{3}\right],
$$

where $\delta$ is a function of $x$ only. Recall that $h$ is a function of $t$ only. Differentiating equation (64) with respect to $x$ and using equations (21) and (22) gives

$$
\begin{aligned}
3 \alpha \delta y^{2}+ & \left\{\delta_{x}+3 \beta \delta+2 \alpha\left[\left(\beta^{2}+2 \alpha \gamma\right) x-\beta\right]\right\} y \\
+ & \gamma\left\{2 x \alpha_{x}+3[\alpha+\delta]\right\}=0
\end{aligned}
$$

for all $t$. Since $y$ must have non-constant $t$-dependence, the coefficients of different powers of $y$ in equation (65) must vanish identically. If $\alpha=0$ then $g=0$ and there are no Riccati solutions. Therefore the coefficient of $y^{2}$ in equation (65) shows that $\delta$ is identically zero. The constant term in equation (65) shows that either $\gamma \equiv 0$ or $\alpha(x)=\kappa x^{-3 / 2}$, where $\kappa$ is a constant.

If $\gamma=0$ then the coefficient of $y$ in equation (65) shows that either $\beta=0$ or $\beta=1 / x$. These solutions correspond to the solutions (25) and (26) respectively. Finally, if $\gamma \not \equiv 0$ and $\alpha \not \equiv 0$ then recall from section 2 (case 3) that for any Riccati solution we must have $\alpha=-\left(\gamma^{-1}\right)_{x x}$ and $\beta=-\gamma_{x} / \gamma$. It follows that the coefficient of $y$ and the constant term in equation (65) cannot both vanish identically.

\section{Bäcklund transformations and special solu- tions}

In this section we will construct what is perhaps the simplest solution of equation (1) involving a genuine transcendent of the second Painlevé equation. It is simple in the sense that we have an explicit formula for the dependence of $x$ on $z$. We will then use the well-known Bäcklund transformation of the second Painlevé equation to construct a countable family of equations of the form (1) together with their general solutions in terms of second Painlevé transcendents.

If $a \neq 0$ then, after rescaling $z$ and $v$, equations (14-15) become

$$
\frac{d^{2} v}{d z^{2}}=2 v^{3}+z v+\alpha
$$




$$
\frac{d^{2} w}{d z^{2}}=\left(6 v^{2}+z\right) w
$$

where $\alpha$ is an arbitrary constant. Equation (66) is the standard form of the second Painlevé equation. We will denote the general solution of equation (66) by $v(z)=P_{I I}\left(z ; \alpha ; c_{1}, c_{2}\right)$, where $c_{1}$ and $c_{2}$ are independent parameters (e.g. $c_{1}=v(0)$ and $\left.c_{2}=v^{\prime}(0)\right)$.

Recall that, apart from the solution due to Shah and Vaidya (equation 28), many of the solutions that appear in the literature are special cases of the solutions of Sussman [15], which correspond to the special case of proposition 1.1 in which $a=0$ and $v$ is a constant. Note that if $a \neq 0$, then equation (66) (which is a rescaled version of equation 14) admits a constant solution if and only if $\alpha=0$. In this case the constant solution is $v \equiv 0$, which is equivalent to the case $f \equiv 0$.

If $v \equiv 0$ then equation (67) has the general solution

$$
w(z)=\mu \operatorname{Ai}(z)+\nu \operatorname{Bi}(z),
$$

where $\mathrm{Ai}$ and $\mathrm{Bi}$ are the Airy functions and $\mu$ and $\nu$ are arbitrary constants which are not both zero. From equation (12) we have

$$
x=\pi \frac{\rho \operatorname{Ai}(z)+\sigma \operatorname{Bi}(z)}{\mu \operatorname{Ai}(\mathrm{z})+\nu \operatorname{Bi}(\mathrm{z})},
$$

where $\rho$ and $\sigma$ are arbitrary constants satisfying $\mu \sigma-\nu \rho=1$. (Note the identity $\operatorname{Ai}(z) \operatorname{Bi}^{\prime}(z)-\operatorname{Bi}(z) \operatorname{Ai}^{\prime}(z)=\pi^{-1}$.) In particular, choosing $\mu=\sigma=1$ and $\nu=\rho=0$, we see that the general solution of $y_{x x}=\mathrm{Bi}^{6}(z) y^{3}$ is

$$
y(x)=\frac{P_{I I}\left(z ; 0 ; c_{1}, c_{2}\right)}{\operatorname{Bi}(z)},
$$

where $c_{1}$ and $c_{2}$ are arbitrary constants (or functions of $t$, viewing the equation as a $\mathrm{PDE}$ ) and $z$ is given by

$$
\frac{\operatorname{Bi}(z)}{\operatorname{Ai}(z)}=\frac{x}{\pi} .
$$

Now we will see how to generate other solutions from the $v=0$ case just described. Let $v$ be a solution of equation (66) where $\alpha \neq-1 / 2$. Then it is well known [21] that

$$
\tilde{v}:=-v-\frac{1+2 \alpha}{2 v_{z}+2 v^{2}+z}
$$


satisfies equation (66) with $\alpha$ replaced by $\alpha+1$. Equation (68) is the standard Bäcklund transformation of equation (66). Let $V(z ; \epsilon)$ be a one-parameter (i.e. $\epsilon$ ) family of solutions to equation (66). Since equation (67) is the linearization of equation (66), it follows that $W(z ; \epsilon):=V_{\epsilon}(z ; \epsilon)$ is a solution to equation (67). Substituting $v=V(z ; \epsilon)$ into equation (68) and differentiating with respect to $\epsilon$ shows that

$$
\tilde{w}:=-w+2(1+2 \alpha) \frac{w_{z}+2 v w}{\left(2 v_{z}+2 v^{2}+z\right)^{2}}
$$

satisfies equation (67) with $v$ replaced by $\tilde{v}$, whenever $w$ satisfies equation (67).

Applying the Bäcklund transformations (68-69) to $v(z)=0, w(z)=$ $\operatorname{Ai}(z)$, described above, yields $\tilde{v}(z)=-z^{-1}$ and $\tilde{w}(z)=2 z^{-2} \operatorname{Ai}^{\prime}(z)-\operatorname{Ai}(z)$. It follows that

$$
y(x)=\frac{z^{2} P_{I I}\left(z ; 1 ; c_{1}, c_{2}\right)+z}{2 \operatorname{Ai}^{\prime}(z)-z^{2} \operatorname{Ai}(z)}
$$

is the general solution of equation (1) with $f(x)=6 \tilde{v}(z) \tilde{w}^{5}(z)$ and $g(x)=$ $2 \tilde{w}^{6}(z)$, where

$$
\frac{2 \operatorname{Bi}^{\prime}(z)-z^{2} \operatorname{Bi}(z)}{2 \operatorname{Ai}^{\prime}(z)-z^{2} \operatorname{Ai}(z)}=\frac{x}{\pi}
$$

Repeated application of the Bäcklund transformations (68-69) will generate a countable family of equations of the form (1) and solutions in which $v$ is a rational function of $z$ and $w$ is a rational function of $z$, the Airy functions $\mathrm{Ai}$ and $\mathrm{Bi}$ and their first derivatives.

\section{Discussion}

The search for metrics modelling non-static shear-free spherically symmetric charged fluids naturally leads to the problem of finding one-parameter families of solutions to equation (1). The Painlevé property is a very powerful detector of the integrability of ODEs. Indeed, most of the solutions to equation (1) that have appeared in the literature to date arise naturally from the Painlevé analysis of equation (1) (see [14]). However, since equation (1) is second-order while we only require a one-parameter family of solutions, it is not necessary for us to describe the general solution. From this point of view, requiring the Painlevé property is too restrictive. 
In this paper we have considered the problem of determining one-parameter families of solutions to equation (1) whose only movable singularities are poles. Besides the solutions covered by proposition 1.1 (which corresponds to the cases in which equation 1 possesses the full Painlevé property) we found one-parameter families of solutions that satisfy Riccati equations. In particular, this class of solutions contains those of Shah and Vaidya, which do not arise in the standard Painlevé analysis of equation (1). The procedure for matching the Riccati solutions to an external Reissner-Nordstrøm metric was also described.

Finally, a special sub-class of solutions that arise in proposition 1.1 were described. In general, when $a \neq 0$, the transformation between $x$ and $z$ involves derivatives of a second Painlevé transcendent. In the class of solutions described in section $6, v, w$, and $x$ are given explicitly in terms of Airy functions and there first derivatives - only $u$ is a genuine Painlevé transcendent. Presumably this is the simplest class of solutions characterized by proposition 2 that contains a genuine Painlevé transcendent.

\section{Acknowledgements}

This work was supported by the Royal Society (research grant number 22204).

\section{References}

[1] Y.P. Shah and P.C. Vaidya. Gravitational field of a charged particle embedded in a homogeneous universe. Tensor N. S. 19, 191-194 (1968).

[2] M.C. Faulkes. Charged spheres in general relativity. Can. J. Phys., 47, 1989-1994 (1969).

[3] A. Krasiński. Inhomogeneous Cosmological Models. CUP, Cambridge, (1997).

[4] S. Kowalevski. Sur le problème de la rotation d'un corps solid autour d'un point fixè. Acta Math., 12, 177-232 (1889). 
[5] S. Kowalevski "Sur une propriété d'un système d'équations différentielles qui definit la rotation d'un corps solide autour d'un point fixè", Acta Math., 14, 81-93 (1889).

[6] E.L. Ince, "Ordinary Differential Equations", Dover, New York (1956).

[7] M.J. Ablowitz and P.A. Clarkson. Solitons, Nonlinear Evolution Equations and Inverse Scattering, Lond. Math. Soc. Lecture Note Series vol. 149. Cambridge University Press, Cambridge (1991).

[8] H. Flaschka and A.C. Newell. Monodromy- and spectrum preserving deformations. I. Commun. Math. Phys. 76, 65-116 (1980).

[9] M. Wyman. Non-static radially symmetric distributions of matter. Canad. Math. Bull. 19, 343-357 (1976).

[10] M. Wyman. Nonstatic spherically symmetric isotropic solutions for a perfect fluid in general relativity. Aust. J. Phys. 31, 111-114 (1978).

[11] S. Chatterjee. Nonstatic charged fluid spheres in general relativity. Gen. Relativity Gravitation, 16, 381-385 (1984).

[12] S.D. Maharaj, P.G.L. Leach, and R. Maartens. Expanding Spherically Symmetric Models without Shear. Gen. Relativity Gravitation 28, 35-50 (1996).

[13] D.C. Srivastava. Exact solutions for shear-free motion of spherically symmetric charged perfect fluid distributions in general relativity. Fortschr. Phys. 40, 31-72 (1992).

[14] R. Halburd. Solvable models of relativistic charged spherically symmetric fluids. Class. Quantum Grav. 18, 11-25 (2001).

[15] R. Sussman. On spherically symmetric shear-free perfect fluid configurations (neutral and charged) I. J. Math. Phys. 28, 1118-1136 (1987).

[16] Y.P. Shah and P.C. Vaidya. Gravitational contraction of charged fluid spheres. Ann. Inst. Henri Poincaré 6, 219-224 (1967).

[17] L. Fuchs. Über Differentialgleichungen deren Intégrale feste Verzweigungspunkte besitzen. Sitz. Akad. Wiss. Berlin 32, 669-720 (1884). 
[18] Y.M. Chiang and R.G. Halburd. On the meromorphic solutions to an equation of Hayman. Submitted (2001).

[19] G. Jank and L. Volmann, "Einführung in die Theorie der ganzen und meromophen Funktionen mit Anwendungen auf Differentialgleichungen", Birkhäuser, Basel-Boston (1985).

[20] E. Hille, Ordinary Differential Equations in the Complex Domain, Wiley, New York (1976).

[21] B. Gambier. Sur les équations différentielles du second ordre et du premier degre dont l'intégrale générale est à points critiques fixés. Acta Math. 33, 1-55 (1909). 